\title{
Cost Analysis of ComFrame: A Communication Framework for Data Management in Mobile Location Based Services
}

\author{
R.Gobi ${ }^{1}$, N.Arulkumar ${ }^{2}$, Dr.E.Kirubakaran ${ }^{3}$, Dr.E.George Dharma Prakash Raj ${ }^{4}$ \\ ${ }^{1}$ Research Scholar, School of Computer Science and Engineering, Bharathidasan University, Trichy-23. \\ ${ }^{2}$ Assistant Professor, Department of Computer Science, Bishop Heber College, Trichy-17. \\ ${ }^{3}$ Additional General Manager, Outsourcing Department, Bharat Heavy Electricals Limited, Trichy-14. \\ ${ }^{4}$ Assistant Professor, School of Computer Science and Engineering, Bharathidasan University, Trichy-23.
}

\begin{abstract}
The main focus of cost analysis is to reduce the operation and reporting of each issue one by one. This includes an idea to improve the efficiencies in data management. It is used to evaluate the desirability of a particular model. It helps to forecast whether the benefits of a framework or a model be more important than its cost. This paper involves in a study of costs and related economic implications that comprise a generic Costbenefit Analysis in ComFrame. ComFrame is a communication framework fashioned to support for data management perspective in mobile location based services.
\end{abstract}

Keywords: Mobile Location Based Services, Cost-Benefit Based analysis and Dynamic data management

\section{Introduction}

A practice of gathering data in a manufacturing atmosphere is to find out the cost of developing a model which is more useful to the mobile user or client. This includes the design phase, buying equipments and materials, production of the hardware and software, etc. A cost analysis is a complete chart of the potential danger and profits of a predictable design to implement any models like ComFrame. Many issues are involved, including a few abstract reflections, making the creation of design and evaluating the design a framework like ComFrame based on cost analysis. The user may change their location at any time and their data access is also the dynamic one which is difficult to manage.

Each analysis has different parameters and demand careful and innovative thought. There is no 'cookbook' for cost-benefit based analysis for a particular framework like ComFrame in Mobile Location Based Services.

II. RELATED WORK: ComFrame

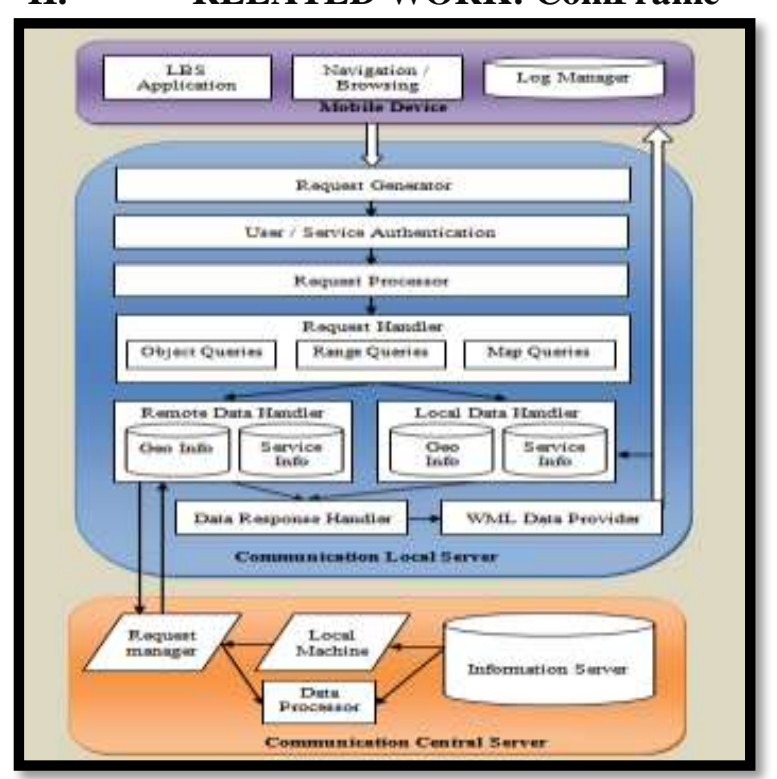

Figure 1: Communication Framework for LBS

The above figure 1 presents communication framework for data management in mobile LBS which consists of Mobile Device, Communication Local Server and Communication Central Server. To resolve the data management issues in mobile computing environment, we developed a communication framework as a solution for dynamic issues in LBS. The framework has been designed with several principles to replicate the 
existing and predictable future condition of the wireless communication technologies. It has several advantages when it is considered for practical usages which were discussed detail in our earlier work [1].

\section{Cost Analysis}

Interest in cost analyses has come with concern about rising hardware and software cost, stress on allocating resources to the users, and the need for product manufacturer and other technology advocates to exhibit the economic benefits of their technologies. This significance is a sign of considerable rise in the number of reports of cost literature and further refinement of methods.

There is a variety of approaches to cost analysis, the correctness of any of which depends upon the function of an evaluation and availability of data and other resources. It is rarely possible to categorize and calculate all costs and all benefits, and the entities used to compute these may differ.

The cost analysis could be classified as the following categories:

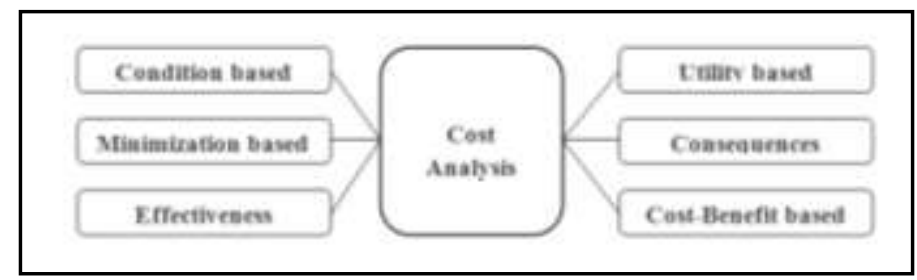

Figure 1: Classification of Cost Analysis

- The Condition based analysis engages the purpose of the economic impact of a circumstance on a given user and the location of a user in location based services.

- The Minimization based analysis occupy in shaping the least costly among alternative interventions that are assumed to generate equivalent conclusions.

- The Effectiveness based analysis bear out the comparison of costs in financial units with results in quantitative non financial units.

- The Utility based analysis confer us a form of cost-effectiveness analysis that compares costs in financial units with results in terms of their utility.

- The Consequence based analysis gives us a form of cost-effectiveness studies that presents costs of the machines and outcomes in discrete categories without comprehensive or weighting them.

- The Cost-Benefit based analysis evaluates costs and benefits, both of which are quantified in common economic entity.

\subsection{A Cost-Benefit based Analysis}

It is valuable for building any decision, whether business or personal, particularly in any case where it is important to build out the risk factor before proceeding. Conducting a cost analysis can be a difficult task in the field of mobile location based services. The component of Cost-Benefit based analysis might be included with four components. They are:

a. Parameter that are considered in a Table

b. Incremental effects model should be shown

c. Table of cost and benefits over the past to present time

d. Statistical and graphical results to show the risks involved in the framework

The general flow of the Cost-Benefit based analysis is shown in Figure 2.

There are different parameters are used to find out the Costs and Benefits. The one must choose right parameters for the analysis.

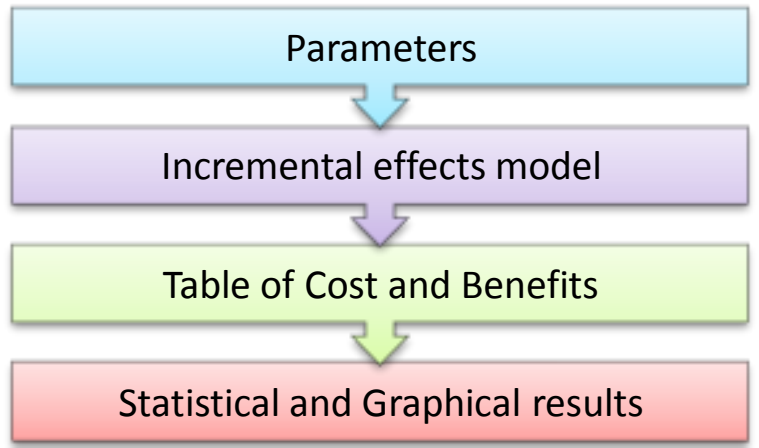

Figure 2: Flow of Cost-Benefit based analysis

The Cost-Benefit based analysis is carried out by considering the following: 
1. Listing out the different projects/programs and stakeholders. Find out the needs, formulate objectives and targets

2. Picking up the measurement(s) and evaluating all cost and benefits essentials

3. Investigate incremental effects and collect information about costs and benefits. Manipulate the results over relevant time phase

4. Changing all costs and benefits into a general currency

5. Investigate risk about the ranges and probabilities by replicating expected outcomes of the investment. Apply discount rate

6. Finding Net Present Value(NPV) of project choices

7. Completing sensitivity analysis

8. Approving recommended choice

This sequence is the preferable way to construction the Cost-Benefit based analysis report.

\subsection{Evaluation based on Cost-Benefit based Analysis} following:

The Cost-Based Analysis attempts to figure out the pros and cons of framework which includes the

a. Special outcome on mobile users

b. Effects on non-mobile users

c. Externality causes on clients and servers

d. Option value or any social issues

\subsection{Overlooked Costs}

The typical failure of a Cost-Benefit based analysis does not cover all the expenditures. In case of the communication local and Central Server, we have some of overlooked cost. They are:

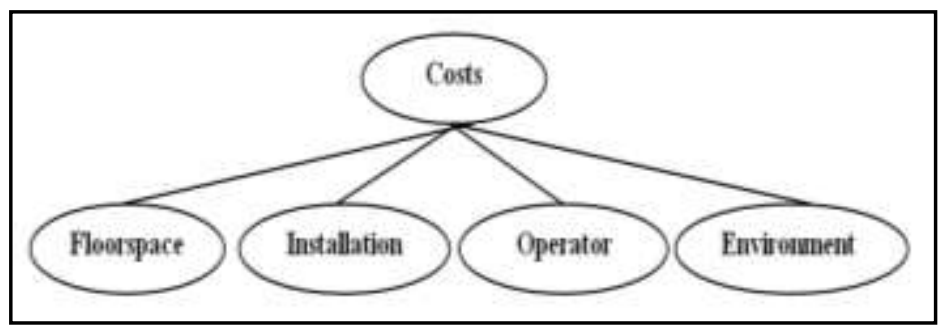

Figure 3: Overlooked Costs

Floor Space: It defines how the client server and central server will fit in the same work space which is currently occupied by the machines and workers.

Installation Costs: It defines the cost to eliminate the manual work and to install the new server. At some time, the specialized persons or machinists with extra skills is needed to install the hardware and software. The costs should fit into the estimated level.

Operator Costs: It relates to the work are all involved in the operating the machines. The verification should be done whether they need any special guidance to carry out the work allotted. It includes the worker's salary, fixed charge, expenditure, etc,

Environment Costs: It tells us, if the installed computers might be so noisy and special steps to be carried out around it like fixed soundproofing.

\section{System Analysis}

Scenario 1: BCA measures: Several variations on the basic benefit-cost rule can be used to compare the benefits and costs of investments, projects, or decisions. After collecting all the factors that relates to analysis, the data's are grouped together to calculate accurate Cost-Benefit analysis.

\begin{tabular}{|r|r|r|r|r|r|r|r|}
\hline \multicolumn{7}{|c|}{ Table 1: Comframe : Cost-Benefit Based Analysis } \\
\hline Year & $\begin{array}{c}\text { Installa } \\
\text {-Tion Costs }\end{array}$ & $\begin{array}{c}\text { Operating } \\
\text { Costs }\end{array}$ & Benefits & $\begin{array}{c}\text { Total } \\
\text { Costs }\end{array}$ & $\begin{array}{c}\text { Total } \\
\text { Benefits }\end{array}$ & \multicolumn{1}{c|}{ Discount } & Present \\
\hline 0 & $\$ 10,000.00$ & $\$ 0.00$ & $\$ 0.00$ & $\$ 10,000.00$ & $(\$ 10,000.00)$ & 1.00 & $(10,000.00)$ \\
\hline 1 & $\$ 0.00$ & $\$ 9,500.00$ & $\$ 14,000.00$ & $\$ 9,500.00$ & $\$ 4,500.00$ & 0.91 & $4,090.91$ \\
\hline 2 & $\$ 0.00$ & $\$ 10,000.00$ & $\$ 15,000.00$ & $\$ 10,000.00$ & $\$ 5,000.00$ & & $4,132.23$ \\
\hline
\end{tabular}


A21250 Cost Analysis of ComFrame: A Communication Framework for Data Management in Mobile

\begin{tabular}{|r|r|r|r|r|r|r|r|}
\hline \hline 3 & $\$ 0.00$ & $\$ 11,000.00$ & $\$ 16,000.00$ & $\$ 11,000.00$ & $\$ 5,000.00$ & 0.75 & $3,756.57$ \\
\hline 4 & $\$ 0.00$ & $\$ 12,000.00$ & $\$ 17,000.00$ & $\$ 12,000.00$ & $\$ 5,000.00$ & 0.68 & $3,415.07$ \\
\hline 5 & $\$ 0.00$ & $\$ 13,000.00$ & $\$ 18,000.00$ & $\$ 13,000.00$ & $\$ 5,000.00$ & 0.62 & $3,104.61$ \\
\hline 6 & $\$ 0.00$ & $\$ 14,000.00$ & $\$ 19,000.00$ & $\$ 14,000.00$ & $\$ 5,000.00$ & 0.56 & $2,822.37$ \\
\hline 7 & $\$ 0.00$ & $\$ 15,000.00$ & $\$ 20,000.00$ & $\$ 15,000.00$ & $\$ 5,000.00$ & 0.51 & $2,565.79$ \\
\hline 8 & $\$ 0.00$ & $\$ 16,000.00$ & $\$ 21,000.00$ & $\$ 16,000.00$ & $\$ 5,000.00$ & 0.47 & $2,332.54$ \\
\hline
\end{tabular}

The usual analysis is done by finding the difference between, the addition of all the benefits, and by grouping all the cost factors. The method which is considered here is an easiest one for reviewing our work in the future and it is done by grouping all the factors together. Cost-Benefit based analysis is prepared for one month to purchase new machines like central server and local server. It proves the purchase of the new server is reasonable. The machine will save the costs each and every month.

The above table defines the standard cost/benefit criteria are incorporated in spreadsheet program. The data in the table shows cost and benefit details if ComFrame is installed and it is made for public use to generate revenue. At first, the model is installed at a cost $\$ 10,000$ at year 0 . The company gets benefit from the subsequent year 1 to year 8 . The graph shows that there is increased revenue from the year 1 . To implement graph, the parameters chosen are year against to total costs and total benefits.

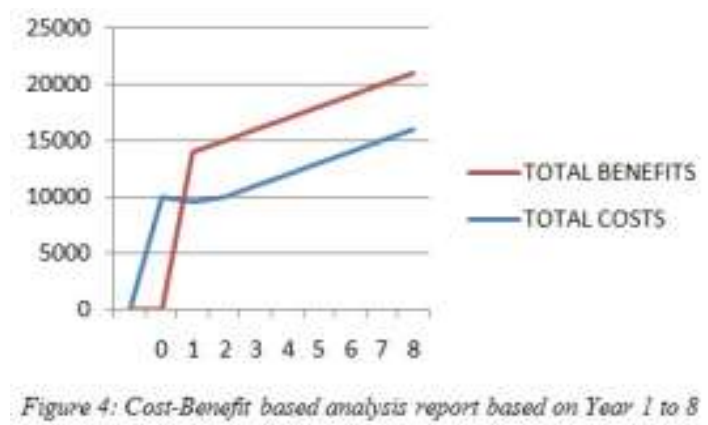

The information is the stream of costs and benefits (in millions) estimated for a proposed model like ComFrame on the basis of dynamic data management in mobile location based services. Year 0 represents the initial investment while costs for years 1-8 are the maintenance costs incurred at the end of each year. The benefits are the revenues from company team contracts and revenues at the end of each year.

Net present value (NPV)

The net present value (NPV) is the current value of all project net benefits. Net benefits are simply the sum of benefits minus costs. The formula used to calculate the NPV is:

$$
\mathrm{NPV}=\sum_{t=1}^{T} \frac{\left(\text { Benefit }_{t}-\text { Cost }_{t}\right)}{(1+r)^{t}}
$$

The sum is discounted at the discount rate. Using this method, if the project has a NPV greater than zero then it appears to be a good candidate for implementation. Based on this formulae, we have achieved the $N P V=\$ 16,220.09$

Benefit-cost ratio (BCR)

The benefit-cost ratio (BCR) is calculated as the NPV of benefits divided by the NPV of costs:

$$
B C R=\frac{\sum_{t=1}^{r} \frac{B_{t}}{(1+r)^{t}}}{\sum_{t=1}^{T} \frac{C_{t}}{(1+r)^{t}}}
$$

Here, $B_{t}$ is the benefit in time $t$ and $C_{t}$ is the cost in time $t$. If the BCR exceeds one, then the project might be a good candidate for acceptance. The discount rate applied here is $10 \%$.

Internal rate of return (IRR)

The internal rate of return (IRR) is the maximum interest that could be paid for the project resources, leaving enough money to cover investment and operating costs, which would still allow the investor to break even. In other words, the IRR is the discount rate for which the present value of total benefits equals the present value of total costs:

$$
P V(\text { Benefits })-P V(\text { Costs })=0 \text {. }
$$


In general, the IRR should be greater than the discount rate for a project to be accepted. In Excel, the net present value function is = NPV and the internal rate of return function is = IRR. Based on the above table we have got the IRR as $46 \%$.

Scenario 2 - Calculating the discount rate for any particular service used: The discount rate calculation is used to implement any model or any framework is carried out by compiling the discounted stream of costs (or benefits) over time.

$$
\mathrm{p}=\sum_{k=0}^{k} \frac{F k}{(1+r)^{k}}
$$

Here, $\mathrm{p}$ is the present value, $\mathrm{f}$ is the future cost (or benefits) at year $\mathrm{k}$ and $\mathrm{r}$ is the annual discount rate given to the customer when they try to access a service based on the ComFrame for mobile Location Based Services. The main advantage of using this formulae is to find out the discount rate given to customers for any particular service used like application cost, system utilization charge, etc.,

Example: The present value of a cost (or benefit) of $\$ 1,000$ It determines the present value of future costs and benefits. In the first year, using the $10 \%$ discount rate, is $\$ 910$.

Scenario 3 - Calculating a GetBack time for ComFrame: Considering the cost and benefit list into account, the amount of time is taken which will recover the projected costs. Example: If we need to implement a local server which will interact with mobile user at a cost of 10000 dollars, with a projected profit of 5000 dollars per month, then our regain time is 2 months.

\section{Advantages of Cost Benefit Analysis in ComFrame:}

The cost analysis is carried out in ComFrame to increase the cost by implementing mobile location based client and server, to reduce the maintenance cost and to manage the integration cost which includes several issues. The other advantages includes

1. Money saved

2. profits gained

3. Time and effort saved

4. Interest accrued

5. Equity built

Benefits: The Cost-Benefit based analysis is carried out to support for clients and also for service providers and site administrators. The company can use the model to predict their benefits before they implement the framework like ComFrame. The discount rate is applied and it supports for the customer to get benefits.

Point to keep in mind: The general points should be kept in mind while introducing Cost-Benefit analysis for frameworks like ComFrame. They are:

1. Create an environment of trust

2. Adapt the instruction of the analysis

3. Ensure the service or procedures reflects appropriate, safe practices

4. Cut costs by eliminating unnecessary efforts and prevent rework and waste

Note: There are some dangers of cost-cutting measures. In order to save money, that leads to reduce number of staffs and materials. So, it leads to extra work for the employees and it makes client for long waiting to receive the products which are such a good quality.

\section{Conclusion}

In simple words, Cost-Benefit analysis is referred as a process of maximizing benefits and minimizing costs. A smart way of establishing a unit of measurement is used throughout the measurement. Here, the predominate unit of measurement considered are cost and benefits.

The Cost-Benefit based analysis is the de facto standard for representing financial possibility to implement any model. It also does the comparison and to perform selection among the investment in that particular field. The main necessity of the Cost-Benefit based analysis is to perform some analysis and to present some data related to risk management, costs and benefits of a given framework like ComFrame so that it can be compared to any other investment opportunities.

\section{References:}

[1] R.Gobi, Dr.E.Kirubakaran and Dr.E.George Dharma Prakash Raj, " ComFrame: A Communication Framework for Data Management in Mobile Location Based Services", IJCST, Vol. 3, Issue 7, July 2012.

[2] Daniel Barbará, "Mobile Computing and Databases -A Survey", IEEE Transactions on Knowledge and Data Engineering, VOL. 11, NO. 1, JANUARY/FEBRUARY 1999

[3] Ana M. Bernardos, José R. Casar and Paula Tarrío, " Building a framework to characterize location-based services" International Conference on Next Generation Mobile Applications, Services and Technologies (NGMAST 2007), IEEE Computer Society 2007

[4] Klaus Rechert, " MobIS: A Pragmatic Framework for Location Based Services" Workshop on Positioning, Navigation and Communication IEEE 2009 
[5] F.M. Dahunsi,B. Dwolatzky, " Conceptual Framework that Supports Environment-Aware Positioning and Improved QoS for Location Based Services", IEEE AFRICON 2009

[6] Chulbum Ahn and Yunmook Nah, "Design of Location-based Web Service Framework for Context-Aware Applications in Ubiquitous Environments" IEEE Computer Society 2010

[7] Hanunah Othman, Habibah Hashim, Mohd Ameer Yuslan Razmi, Jamalul-lail Ab Manan, " Forming Virtualized Secure Framework for Location Based Services (LBS) using Direct Anonymous Attestation (DAA) protocol", IEEE 2010

[8] Nimalika Fernando, Dileeka Dias, Sohan Wijesekara"A framework to develop location based services applications using OGC map services", IEEE 2010

[9] Carlos L"ubbe, Andreas Brodt, Nazario Cipriani, Matthias Grosmann, Bernhard Mitschang, " DiSCO: A Distributed Semantic Cache Overlay for Location-based Services" IEEE International Conference on Mobile Data Management 2011

[10] Yu Chen, Jie Bao, Wei-Shinn Ku, Jiun-Long Huang, "Cache Management Techniques for Privacy Preserving Location-based Services", MDM, 2008.

[11] Rechert, K, " MobIS: A pragmatic framework for LBS", Positioning, IEEE 2009

[12] Dahunsi, F.M.; Dwolatzky, " Conceptual framework that supports environment-aware positioning and improved QOS for Location Based Services", IEEE 2009

[13] Bernardos, A.M. , " Building a framework to characterize location-based services", IEEE 2007

[14] Ahn, Chulbum Nah, Yunmook "Design of Location-Based Web Service Framework for Context-Aware Applications in Ubiquitous Environments", IEEE 2010.

[15] Reza Shokri, Julien Freudiger ,Jean-Pierre Hubaux, "A Unified Framework for Location Privacy" EPFL Report June 2010 